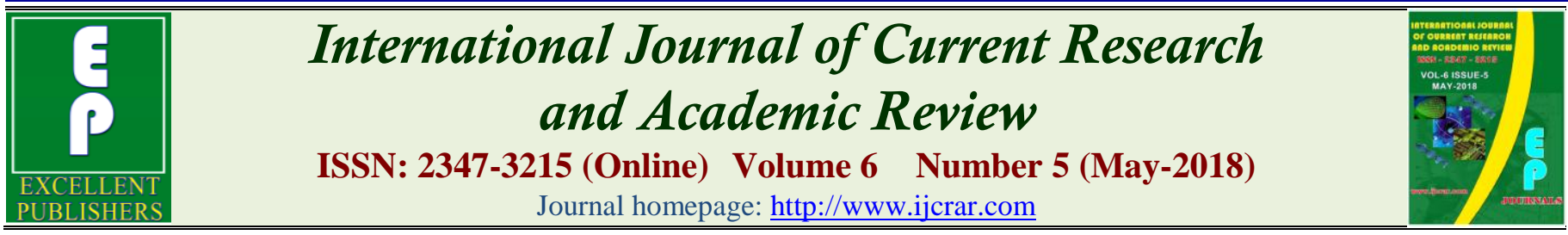

doi: https://doi.org/10.20546/ijcrar.2018.605.002

\title{
L-Arginine, A Nitric Oxide Donor: A Study of Its Effect on Ovarian and Endometrial Vascular Parameters and Fertility Outcome in PCO Infertile Patients
}

\author{
Meenal Jain* \\ Department of OBGYN, S.N.M.C. Agra, India \\ *Corresponding author
}

\section{Abstract}

To study the effect of adjuvant L-arginine treatment on ovarian and endometrial vascular parameters and fertility out come in clomiphene treated PCO infertile patients. 60 PCO patients defined by revised Rotterdam criteria were randomly divided into a control and study group with 30 cases each. Control group was given clomiphene citrate $(50-150 \mathrm{mg})$ for 5 days starting from the $2^{\text {nd }}$ day of cycle. The study group in addition received L-arginine $3 \mathrm{gm}$ per day for the whole cycle. Basal day II doppler parameters (ovarian and endometrial) were measured. They were then called for follicular monitoring starting from day 12. Doppler parameters were then evaluated and mean values calculated. Ovulatory and pregnancy rates (by earliest demonstration of fetal cardiac activity) were also noted. Cases were similar in age, BMI and duration of infertility in both groups. There was a significant improvement in vascular parameters in the study group. Uterine RI $(0.489 \pm 0.284)$, uterine PI $(2.79 \pm 0.031)$, PSV $(14.3 \pm 1.85 \mathrm{~cm} / \mathrm{sec})$, ovarian RI $(0.419 \pm 0.348)$, PI $(1.19 \pm 0.46)$, PSV $(15.48 \mathrm{~cm} / \mathrm{sec})$. No of mature follicles (control $0.81 \pm 0.74$ and study $1.19 \pm 0.76$ ). Endometrial thickness (control $8.1 \pm 1.21 \mathrm{~mm}$ and study $9.26 \pm 1.28 \mathrm{~mm}$ ) the values were statistically significant. The PFBF was also increased (Grade-3, 4 ) in the study group. The ovulatory and pregnancy rate in control group was $43.3 \%$ and $13.3 \%$ respectively. While in study group the ovulatory and pregnancy rate were $66 \%$ and $30 \%$ respectively which are statistically significant. Adjuvant L-arginine treatment decreases ovarian and endometrial blood flow impedance, thus improving the vascularity which inturn increases the ovulatory and pregnancy rates in PCO infertile patients.
\end{abstract}

\section{Article Info}

Accepted: 20 April 2018

Available Online: 20 May 2018

\section{Keywords}
Polycystic ovary,
Nitric oxide donors,
L-arginine,
Clomiphene citrate

\section{Introduction}

Polycystic ovary is a common endocrine disorder accounting for more than $70 \%$ cases of anovulatory infertility. Clomiphene citrate (CC) has been the first line treatment for these cases but $25-40 \%$ either remain anovulatory (CC resistant) on fail to conceive in spite of ovulation (CC failure) after three or more treatment cycles with $100-150 \mathrm{mg} \mathrm{CC}$. It has been seen that in dysovulation - follicular development, maturation, ovulation and implantation are impaired due to hormonal imbalance and vascular impairment of the ovarian and uterine vasculature. Increased vascularization of ovarian follicles occurs in the course of their development and seems to be responsible for the selection and maturation of follicles. Gonadotropins, steroids, prostaglandins and other vasoactive molecules such as nitric oxide are involved in the regulation of ovarian blood flow. (Van 
Blerkom et al., 1997) nitric oxide is a labile diffusible, highly reactive and short lived radical with a half-life time of about 10-12 sec L-arginine acts as a substrate for the production of nitric oxide (NO) either by a constitutive calcium dependent or a proinflammatory cytokine inducible nitric oxide synthase. It forms stable oxidised metabolites (nitrite/nitrate; $\mathrm{NO}_{2} \mathrm{NO}_{3}$ ) detectable in many biological fluids. (De Pace et al., 2001)

NO is responsible for periovulatory vasodilatory modulation of ovarian blood flow thus improving follicular maturation and ovulation. It also has an impedance lowering effect on uterine vascular bed thus improving endometrial receptivity which is required to support the decidualization and implantation process.

This study was thus carried out to test if treatment of PCO infertile patients with CC and L-arginine as a NO donor might improve the ovarian and uterine doppler parameters and thus the pregnancy rates in comparison to treatment with $\mathrm{CC}$ alone.

\section{Materials and Methods}

This study was a randomized controlled trial conducted in the period between June 2015 and May 2016.

The study included 60 PCO infertile pateints divided randomly into a control and study group with 30 cases in each group. The study was accepted by the ethical committee and consent was taken from every patient. Polycystic ovary was diagnosed according to the revised Rotterdam criteria (2003): 1. Oligo/anovulation 2. Clinical \& or biochemical signs of hyperadrogenism. 3. PCO morphology on US examination ( $\geq 12$ follicles of $<10 \mathrm{~mm}$ and /or ovarian volume $>10 \mathrm{ml}$ per ovary). Patients fulfilling atleast 2 out of 3 of these criteria were included for the study

\section{Exclusion criteria}

Age $<18$ years or $>35$ years.

Male causes of infertility.

Tubal causes of infertility.

Other causes of infertility like current untreated pelvic pathology. Sub mucosal uterine fibroid/polyp, pelvic inflammatory disease, uterine malformations, Asherman's syndrome, moderate to severe endometriosis,
H/o chemoradiation.

Hypersenstivity.

Other endocrine abnormalities like hyperprolactinemia, thyroid dysfunction, Cushing's syndrome, congenital adrenal hyperplasia, an adrenal/ovarian tumor.

Patients in both groups were treated with clomiphene citrate in a dose of $50-150 \mathrm{mg} /$ day (initial dose was 50 $\mathrm{mg}$ which was increased to $100 \mathrm{mg}$ and then $150 \mathrm{mg}$ in subsequent cycles) for 5 days starting from the second day of cycle. The study group in addition received $3 \mathrm{gm}$ per day of L-arginine for the whole cycle. Day II basal vascular parameters were recorded in both groups. Mean of values from both sides was obtained. Cases were called for monitoring of ovulation and evaluation of periovulatory ovarian (perifollicular) and endometrial vascular parameters via transvaginal sonography with color doppler on every other day from cycle day 12 . TVS was done with a $6.5 \mathrm{MHz}$ colour doppler system (Logiq 3). For doppler examination patients were studied between 8:00-11:00 hrs to exclude the effects of circadian rhythmicity on blood flow. Maximum U/S energy was $<80 \mathrm{mw} / \mathrm{cm}^{2}$. Colour flow images of the ascending branches of the uterine arteries were sampled lateral to the cervix in a longitudinal plane.

The perifollicular arteries were identified around the follicles $(>1.0 \mathrm{~cm})$ in diameter in the ovarian stroma at the maximum distance from the surface of the ovary. Arteries demonstrating lowest downstream impedance were selected for measurements assuming that these branches directly supply the developing follicles. The mean value of 3 consecutive waveforms on the dominant side was obtained.

Presence of one or more follicles $\geq 18 \mathrm{~mm}$ that disappeared or changed in shape in subsequent scans was considered as evidence of ovulation. Endometrial thickness was also recorded. The follicular circumference was frozen and the perifollicular blood flow (PFBF) was graded based on (Chui et al., 1997) according to this modified grading system ovarian PFBF was represented as Grade $0-0 \%$, Grade $1-1-25 \%$, Grade $2-26-50 \%$, Grade $3-51-75 \%$ and Grade $4-76-$ $100 \%$.

Intercourse was advised in the days following ovulation and cases with a missed period and positive UPT were followed till 7 wks for earliest demonstration of fetal cardiac activity (considered as successful pregnancy 
outcome). Treatment in both groups continued for three cycles or till pregnancy was achieved.

Statistical analysis was done using the Fischer's exact test and $\mathrm{p}$ value $(<0.05 \%)$ was considered significant.

\section{Observations}

The study included 60 infertile an ovulatory women with PCOS who were similar in age, BMI and also in reference to the mean duration of infertility (Table 1).

According to table 2 there is no statistical difference between day II doppler parameters in both groups. Table 3 shows significant improvement in the vascular parameters in L-arginine group during the periovulatory period. The perifollicular arteries show an increase in the peak systolic velocity (PSV $15.48 \mathrm{~cm} / \mathrm{sec}$ ) and a corresponding decrease in RI (0.419) \& PI (1.19). The uterine arteries also show decrease in impedance (RI 0.489 and PI (2.79) and increase in PSV $(16.3 \mathrm{~cm} / \mathrm{sec})$ in the $(\mathrm{CC}+\mathrm{Arg})$ group as compared to $\mathrm{CC}$ alone.

It is also depicted here that all cycles in the $(\mathrm{CC}+\mathrm{Arg})$ group showed mature follicles (i.e. follicles greater than $18 \mathrm{~mm}$ ) reflected by a number greater than 1 , while this is not the case with $\mathrm{CC}$ alone group; also the endometrial thickness is better $(9.28 \mathrm{~mm}$ vs. $8.1 \mathrm{~mm})$. On comparing the PFBF grade (Fig. 1) it can be seen that Grade 3 and 4 flow was seen in nearly 20 (66\%) cases of study group. While only 7 in control group had Grade 3 flow and none Grade 4. Majority of cases $15(50 \%)$ in control had a PFBF of Grade 2 and nearly $30 \%$ had values of Grade 1 $\& 0$ while no case in study group had grade 0 PFBF.

Table 4 depicts the ovulatory rate which is $13(43.3 \%)$ in the control group out of which $4(13.33 \%)$ had a successful pregnancy whereas the study group on the other hand had a better ovulatory rate $20(66 \%)$ out of which $9(30 \%)$ had a successful pregnancy.

The results were statistically significant $(\mathrm{p}<.035)$. There was no significant difference between the side effects in both groups (Fig. 2).

\section{Results and Discussion}

The present study compared the fertility promoting effects of adjuvant L-arginine in clomiphene treated infertile PCOS patients.

In our study the number of mature follicles was significantly higher in the CC+Arg. group. It is seen that in PCO there is a significant difference in intra ovarian and uterine artery hemodynamics, both at the baseline ultrasound scan and during clomiphene induced menstrual cycle compared to women with normal ovaries, due to hormonal imbalance.

L-argnine is converted to no by activation of guyanylate cyclase and increase in cyclic guyanylate monocyclase which leads to dephosphorylation of the myosin light chain and inhibition of vascular smooth muscle contraction and vasodilatation (Anteby et al., 1996).

By virtue of being a vasodilator No increases the permeability of follicular epithelium to plasma proteins. As the follicles mature they become more susceptible to FSH and growth hormones, both hormones promote an increased production of $1 \mathrm{GF}-1$ which plays a critical role in follicular maturation and differentiation of granulosa cells (Tau et al., 1997).

First Kupesic and Kurjak reported that blood flow to the uterine, spiral and ovarian arteries has a predictive value in endometrial receptivity (Kupesic and Kurjak, 1993). Bassil et al., have also demonstrated that the RI values of the ovarian artery are a good indicator of ovarian function and may be used to assess the optimal timing for the beginning of HMG (Bassil et al., 1997).

According to Ramsay et al., uterine blood flow and endometrial perfusion can be increased by administration of no donor (Ramsay et al., 1994). It is the follicular micro-environment of the human oocyte that plays a crucial factor in its developmental competence.

The quality and maturity of the oocyte depends on the intrafollicular levels of oxygen which in turn is directly proportional to the degree of follicular vascularity. In our study there was a decrease in ovarian (perifollicular) artery RI (0.97 to 0.419$)$ and PI (2.99 to 1.19$)$ with the addition of L-arginine.

Also there was a difference in PFBF in both groups suggesting that there may be a difference in egg quality in both groups based on the measurement of the surrogate end point of the ovarian follicle vascularity. Also in our study uterine artery RI (1.04 to 0.489) and PI (3.81 to 2.79) decreased with L-arginine thus increasing endometrial blood flow and thickness $(9.26 \mathrm{~mm})$.

An increase in the ovulation rate (66\% - study group vs. $43.3 \%$ - control group) is seen which coupled with increased endometrial receptivity resulted in an increase 
in pregnancy rate $(30 \%$ - study group vs $13.3 \%$ - control group) $(\mathrm{p}<0.035)$.

Table.1 Patient profile

\begin{tabular}{|l|c|c|c|c|}
\hline & Control $(\mathbf{n}=\mathbf{3 0})$ & Study $(\mathbf{n}=\mathbf{3 0})$ & t-value & p-value \\
\hline Age & $28.4 \pm 3.62$ & $27.13 \pm 4.32$ & 1.2342 & $0.2223^{\mathrm{NS}}$ \\
\hline BMI & $27.27 \pm 2.94$ & $27.07 \pm 3.03$ & 0.2595 & $0.7962^{\mathrm{NS}}$ \\
\hline Mean duration of infertility (yrs) & $3.1 \pm 2.4$ & $3.7 \pm 2.2$ & -1.0094 & $0.317^{\mathrm{NS}}$ \\
\hline Infertility type & & & & \\
\hline Primary & $25.83 \%$ & $27.90 \%$ & & \\
\hline Secondary & $5.17 \%$ & $3.10 \%$ & & \\
\hline
\end{tabular}

NS- Not significant

Table.2 Basal (Day II) colour doppler parameters in both groups

\begin{tabular}{|c|c|c|c|c|}
\hline Doppler Parameters & Control $(n=30)$ & Study $(n=30)$ & t-value & p-value \\
\hline \multicolumn{5}{|l|}{ Ovarian perifolicular } \\
\hline • $\quad$ RI & $1.21 \pm 0.56$ & $0.97 \pm 1.12$ & 1.0498 & $0.2997^{\mathrm{N}}$ \\
\hline PI & $2.81 \pm 0.83$ & $2.99 \pm 0.87$ & -0.8199 & $0.4156^{\mathrm{NS}}$ \\
\hline PSV & $8.45 \pm 1.16$ & $8.99 \pm 1.28$ & -1.7122 & $0.0923^{\mathrm{NS}}$ \\
\hline \multicolumn{5}{|l|}{ Uterine } \\
\hline RI & $0.84 \pm 1.16$ & $1.04 \pm 0.20$ & -0.9306 & $0.3593^{\mathrm{NS}}$ \\
\hline PSV & $9.14 \pm 2.05$ & $8.75 \pm 1.71$ & 0.8617 & $0.3925^{\mathrm{NS}}$ \\
\hline PI & $3.75 \pm 0.66$ & $3.81 \pm 0.94$ & -0.2861 & $0.7759^{\mathrm{NS}}$ \\
\hline
\end{tabular}

NS- Not significant

Mean values of arteries on both sides were taken.

Table.3 Follicular monitoring in both groups

\begin{tabular}{|l|c|c|c|c|}
\hline \multicolumn{7}{|l|}{ Doppler Parameters } & Control $(\mathrm{n}=30)$ & Study $(\mathrm{n}=30)$ & $\mathrm{t}$-value & $\mathrm{p}$-value \\
\hline Ovarian perifolicular & $0.538 \pm 0.0 .8$ & $0.419 \pm 0.348$ & 1.8253 & $0.0473^{*}$ \\
\hline$\bullet \quad$ RI & $1.7 \pm 0.35$ & $1.19 \pm 0.46$ & 4.8327 & $<0.0001^{*}$ \\
\hline$\bullet \quad$ PI & $13.24 \pm 1.45$ & $15.48 \pm 1.39$ & -6.1081 & $<0.0001^{*}$ \\
\hline$\bullet \quad$ PSV & $0.67 \pm 0.22$ & $0.489 \pm 0.284$ & 2.7596 & $0.0079^{*}$ \\
\hline Uterine & $11.2 \pm 1.26$ & $14.3 \pm 1.85$ & -7.5858 & $<0.0001^{*}$ \\
\hline$\bullet \quad$ RI & $3.3 \pm 0.035$ & $2.79 \pm 0.031$ & 59.7456 & $<0.0001^{*}$ \\
\hline$\bullet \quad$ PSV & $0.81 \pm 0.7$ & $1.19 \pm 0.76$ & -1.9621 & $0.0446^{*}$ \\
\hline$\bullet \quad$ PI & $8.1 \pm 1.21$ & $9.26 \pm 1.28$ & -3.6071 & $0.0006^{*}$ \\
\hline
\end{tabular}

NS- Not significant

*- Significant at $\mathrm{p}=0.05$

Mean values were taken on the dominant side on the day of impending ovualtion.

Table.4 Comparison of ovulatory and pregnancy rates in both the groups

\begin{tabular}{|l|c|c|c|c|c|}
\hline \multirow{2}{*}{} & \multicolumn{2}{|c|}{ Control $(\mathrm{n}=30)$} & \multicolumn{2}{c|}{ Study $(\mathrm{n}=30)$} & $\mathrm{p}$-value \\
\cline { 2 - 6 } & No. & $\%$ & No. & $\%$ & \\
\hline Ovulatory rate & 13 & 43.3 & 20 & 66.00 & $<0.035^{*}$ \\
\hline Pregnancy rate & 4 & 13.3 & 9 & 30.0 & $<0.024^{*}$ \\
\hline
\end{tabular}




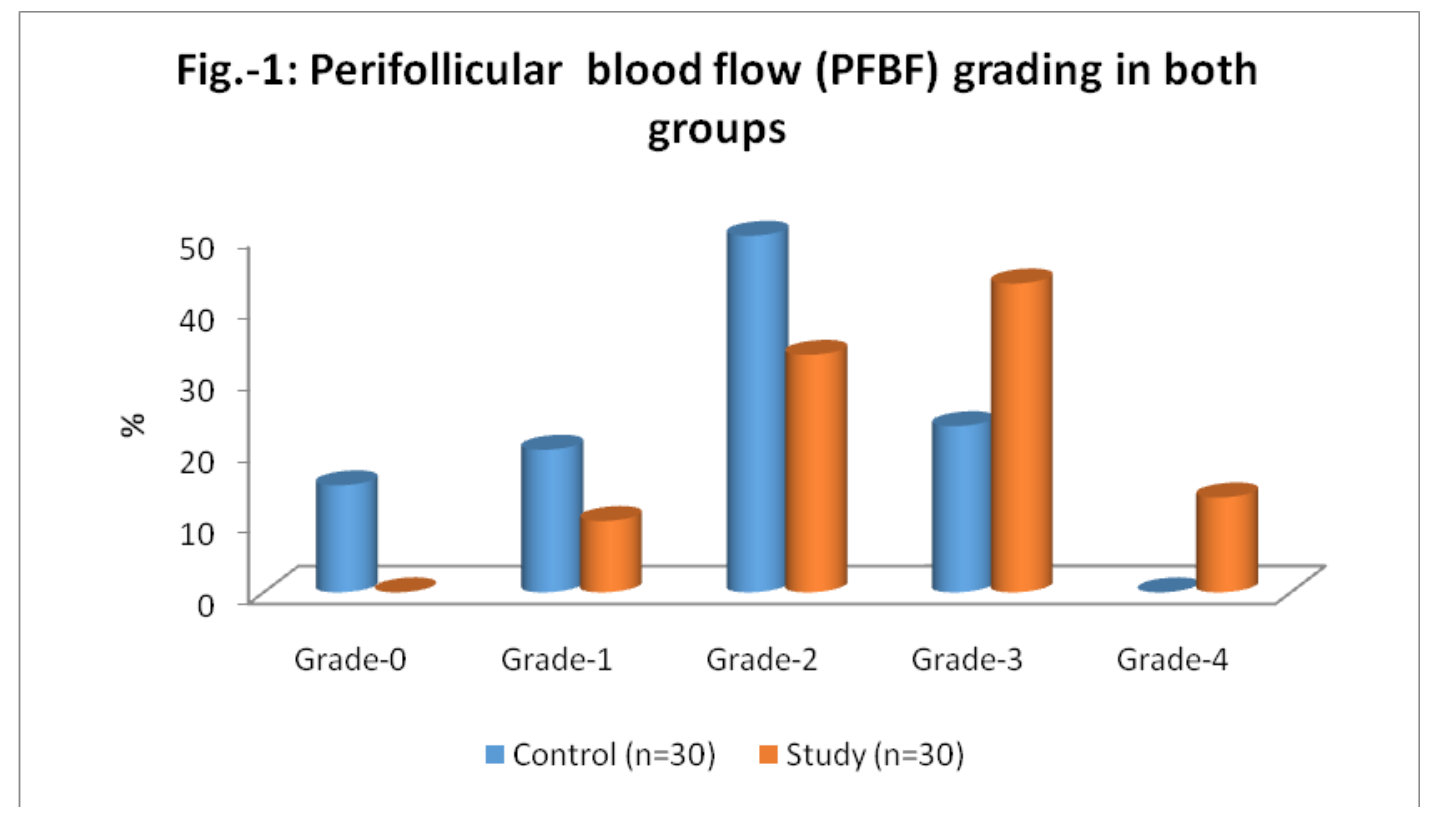

Fig.-2: Side effects recorded during treatment

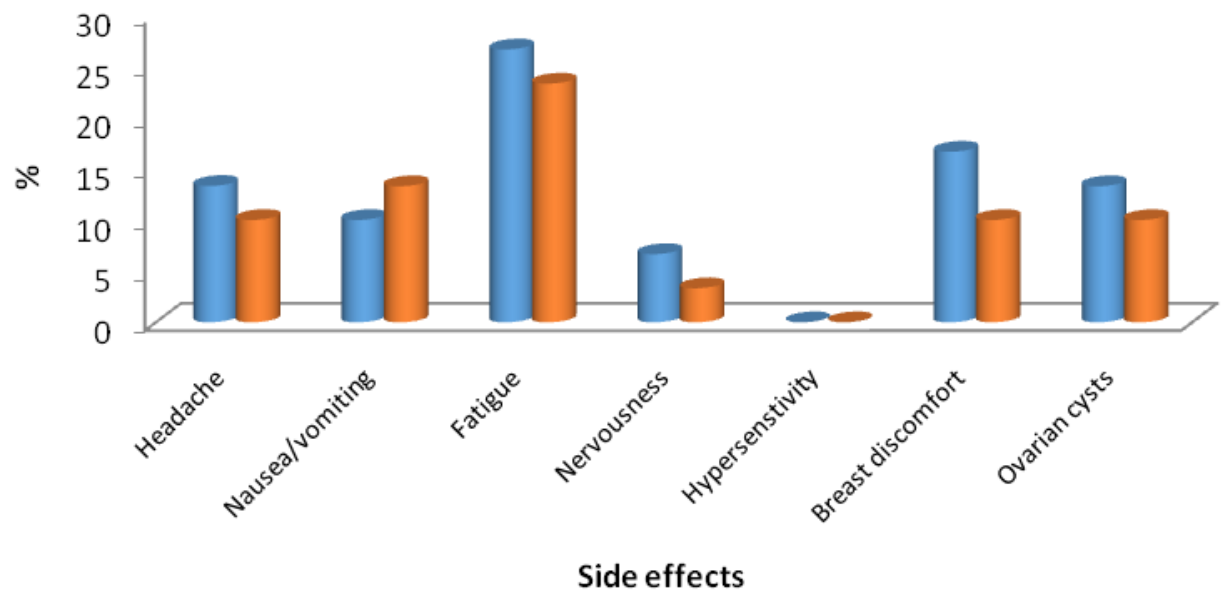

- Control $(n=30) \quad$ Study $(n=30)$

Little information is found in the literature on the use of oral no donors in dysovulatory infertility. One study by (Battaglea Cesare et al., 2002) found that adjuvant oral L- Arginine treatment in poor responders IVF patients decreased RI \& PI of perifollicular and uterine vessels leads to decreased cancellation rate and increased oocyte and embryo transfer.

According to (Seham El Berry Razik Abdel Mohammad, 2010) NO donors (isosorbide mononitrate) increases ovulatory and pregnancy rate in clomiphene citrate treated polycystic ovary infertile patients $(40 \% \mathrm{Vs}$ $59.46 \%)(10 \%$ vs $18.92 \%)$. Better ovulatory and pregnancy rates also seen with study of (Ahmad Mehran et al., 2016)

Our study is different as we have used oral $L$-arginine as a nitric oxide donor for spontaneous stimulated cycles thus making it a cost effective method

Also L-arginine has not been seen to be associated with major side effects of isosorbide mononitrate like nausea, 
flushing, headache etc. thus increasing its patient compliance which might explain the higher pregnancy rate in our study as compared to that by (Seham El Berry Razik Abdel Mohammad, 2010) and (Ahmad Mehran et al., 2016) who have used ISMN as a nitric oxide donor.

Ohi et al., (2002) did not report any doppler changes for ovarian or uterine vessels with the use of nitro glycerine patch that was administered a day before embryo transfer to IVF patients. This might probably be due to the fact that NTG patch was not administered throughout the follicular phase when follicular maturation takes place. Thus it emphasises the administration and significance of L. arginine for enhancing follicular vascularity in the initial phase of follicular maturation.

This novel treatment of dysovulatory infertility with clomiphene and L-arginine is simple and promising. The ovulation and pregnancy rates increase due to improvement of follicular maturation and endometrial receptivity secondary to increased vascular supply. Thus it can be tried in patients of clomiphene citrate failure before shifting to expensive treatments like gonadotropins or laparoscopy. more controlled studies are however required on larger number of patients to support these results.

\section{References}

Ahmad Mehran, Ayman Abdelmezed, Hossan Shoveski et al., Nitric oxide donors improve the ovulation and pregnancy rates in anovulatory women with PCOS treated with clomiphene citrate; ARCT Int. J. of Rep. Biomedicine 2016, Jan. 14(1): 9-14.

Anteby EY, Harwitz A, Korach $\mathrm{O}$ et al., Human follicular nitiric oxide pathway: relationship to follicular size, oestradiol concentrations and ovarian blood flow J Hum Reprod. 1996: 11; 1947-51

Bassil S, Wyns C, Tousant Demyle D, Misolle M, Gordtz S, Donnez J. The relationship between ovarian vascularity and the duration of stimulation in invitro fertilization. J. Hum. Reprod. 1997; 12(6):

\section{How to cite this article:}

Meenal Jain. 2018. L-Arginine, A Nitric Oxide Donor: A Study of Its Effect on Ovarian and Endometrial Vascular Parameters and Fertility Outcome in PCO Infertile Patients. Int.J.Curr.Res.Aca.Rev. 6(5), 7-12.

doi: https://doi.org/10.20546/ijcrar.2018.605.002
$1240-1245$.

Battaglea Cesare, Salvotri Mizhela, Maxia Nicoletta et al., Adjuvant L-arginine treatment in controlled ovarian hyperstimulation. J. Hum. Reprod. 2002; 17(3): 659-665.

Chui DK, Pugh ND, Walker SM, Gregory L, Shaw RW. Folicular vascularity - the predictive value of transvaginal power doppler ultrasonography in and IVF programme. J. Hum. Reprod. 1997; 12: 191-6.

De Pace V, Chiossi G, Facchinetti F. Clinical use of nitric oxide donors and L-arginine in obstetrics, J. Matern Fetal Neonat Med. 2001; 20: 569-579.

Kupesic S, and Kurjak A. Uterine and ovarian perfusion during the periovulatory period assessed by transvaginal colour doppler. J. Fertil. Steril. 1993; 60(3): 439-443.

Ohi J, Lefevvre Maunoury C, Wittemer C et al., Nitric oxide donors for patients undergoing IVF. A prospective, double blind randomized placebo controlled trial. J. Hum. Reprod. 2002; 17(10): 2615-20.

Ramsay B, DeBelder A, Campbell S et al., A nitric oxide donor improves uterine artery diastolic blood flow in normal early pregnancy and in women at high risk of preeclampsia. Eur. J. Clin Invest. 1994; 24: 76-8.

Sehan El Berry, and Razik Abdel Mohammad. Nitric oxide donors increases pregnancy rate in clomiphene citrate treated polycystic ovary infertile patients. Middle East Fertility Society Journal. 2010; 15: 106-109.

Tau M, Kodame H, Kagabu S, et al., Possible contribution of follicular interleukin $1 \beta$ to nitric oxide generation in human preovulatory follicles. J. Hum. Reprod. 1997; 12: 2220-5.

Van Blerkom J. Antezak M. Schroden. The developoment potential of the human oocyte is related to dissolved oxygen content of follicular fluid: Association with vascular endothelial growth factor levels and perifollicular blood flow characteristics. J. Hum. Reprod. 1997; 12: 1047-55. 